Bull. Korean Math. Soc. 49 (2012), No. 5, pp. 1057-1065

http://dx.doi.org/10.4134/BKMS.2012.49.5.1057

\title{
CO-CONTRACTIONS OF GRAPHS AND RIGHT-ANGLED COXETER GROUPS
}

\author{
Jongtae Kim And Myoungho Moon
}

\begin{abstract}
We prove that if $\widehat{\Gamma}$ is a co-contraction of $\Gamma$, then the rightangled Coxeter group $C(\widehat{\Gamma})$ embeds into $C(\Gamma)$. Further, we provide a graph $\Gamma$ without an induced long cycle while $C(\Gamma)$ does not contain a hyperbolic surface group.
\end{abstract}

\section{Introduction}

Let $\Gamma$ be a finite simple graph with the vertex set $V(\Gamma)=\left\{v_{1}, \ldots, v_{n}\right\}$ and the edge set $E(\Gamma)$. Recall that a simple graph is a graph without loops and multiple edges. The right-angled Coxeter group $C(\Gamma)$ on $\Gamma$ is the group with the presentation, in which the generators are $v_{1}, \ldots, v_{n}$ and relators are $v_{1}^{2}, \ldots, v_{n}^{2}$, and $\left[v_{i}, v_{j}\right]$ whenever $e_{\left\{v_{i}, v_{j}\right\}} \in E(\Gamma)$, where $e_{\{u, v\}}$ denotes the only edge connecting the vertices $u$ and $v$. Considering that the right-angled Artin group on $\Gamma$ is the group with the presentation, in which the generators are $v_{1}, \ldots, v_{n}$ and relators are $\left[v_{i}, v_{j}\right]$ whenever $e_{\left\{v_{i}, v_{j}\right\}} \in E(\Gamma)$, the presentation of the right-angled Coxeter group $C(\Gamma)$ has extra relators $v^{2}$ for all $v \in V(\Gamma)$ when it is compared with the presentation of the right-angled Artin group $A(\Gamma)([3])$. $\Gamma$ is called the defining graph of $C(\Gamma)$. By a graph, we mean a finite simple graph throughout the paper.

Motivated by 3-manifold theory, it has been an intriguing question when right-angled Coxeter groups and right-angled Artin groups contain a closed hyperbolic surface group. Many right-angled Artin groups and right-angled Coxeter groups are known to be 3-manifold groups. A right-angled Coxeter group $C(\Gamma)$ contains a closed hyperbolic surface group if $\Gamma$ contains an induced cycle $C_{n}(n \geq 5)([5])$. Similar results for right-angled Artin groups were obtained in [13] and [8]. In fact, a right-angled Artin group $A(\Gamma)$ contains a closed hyperbolic surface group if $\Gamma$ contains an induced cycle $C_{n}(n \geq 5)([13])$ or an

Received June 3, 2011.

2010 Mathematics Subject Classification. Primary 20F36, 20F55, 20F65; Secondary $05 \mathrm{C} 25$.

Key words and phrases. right-angled Artin group, right-angled Coxeter group, hyperbolic surface subgroup.

Supported by Konkuk University in 2004. 
induced anti-cycle $\overline{C_{n}}(n \geq 5)([8])$. Gordon, Long and Reid raised a question whether the converse also holds $([5])$.

Question 3.1 ([5]). Does a right-angled Coxeter group $C(\Gamma)$ contain a hyperbolic surface subgroup if and only if $\Gamma$ contains an induced $n$-cycle for some $n \geq 5$ ?

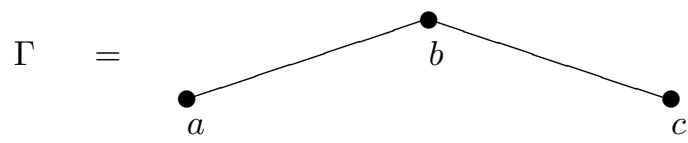

Figure 1. The defining graph of the right-angled Coxeter group with the presentation $\langle a, b, c| a^{2}, b^{2}, c^{2}, a b a^{-1} b^{-1}$, $\left.b c b^{-1} c^{-1}\right\rangle$.

In order to answer the question for the right-angled Artin group case, Kim introduced an operation on a graph, called co-contraction. He proved that if $\Gamma_{2}$ is a co-contraction of a graph $\Gamma_{1}$, then there is a monomorphism from $A\left(\Gamma_{2}\right)$ to $A\left(\Gamma_{1}\right)$. Using this fact, he was able to show that $A\left(\overline{C_{n}}\right)(n \geq 5)$ contains a closed hyperbolic surface group but $\overline{C_{n}}$ does not contain any cycle of length $\geq 5$, thereby gave a negative answer to the question. On the other hand, Bell gave a new proof on Kim's co-contraction lemma on right-angled Artin groups using classical methods from combinatorial group theory ([2]). Bell's method enables us to prove co-contraction theorem for right-angled Coxeter groups as well (see Theorem 2.5). It should be noted that Kim proved Theorem 2.5 for graph products of the cyclic groups of order $m(0<m \leq \infty)$, which includes the right-angled Artin groups and the right-angled Coxeter groups in his dissertation ([7]). But the proof we give is different from than his.

In Section 2, we define co-contractions on graphs and go over Bell's method. Applying Bell's method to right-angled Coxeter group, we prove Theorem 2.5. In Section 3, as an application of Theorem 2.5, we give a negative answer to Question 3.1:

Corollary 3.3. There is a right-angled Coxeter group $C(\Gamma)$ containing a hyperbolic surface subgroup while its underlying graph $\Gamma$ does not contain any induced $n$-cycle for $n \geq 5$.

At the end of Section 3, using the results of Davis, Januszkiewicz [4] and Kim $[7,8]$, we provide examples of different types which justify Corollary 3.3, thereby answer negatively to Question 3.1 again.

\section{Co-contractions of graphs and right-angled Coxeter groups}

A graph is a 1-dimensional CW-complex. 0-cells are called vertices and closed 1-cells are called edges. We denote the vertex set of a graph $\Gamma$ by $V(\Gamma)$ 
and the edge set of a graph $\Gamma$ by $E(\Gamma)$. A loop of a graph is an edge that is homeomorphic to the circle. A graph with multiple edges is a graph that contains two distinct edges sharing their two end points. A simple graph is a graph without loops and multiple edges. In this paper, by a graph we always mean a finite simple graph. Note that every edge is uniquely determined by its two end points in a finite simple graph. We denote the unique edge connecting two adjacent vertices $a, b$ in a graph by $e_{\{a, b\}}$.

Recall that a subgraph $\Gamma^{\prime}$ of a graph $\Gamma$ is called an induced subgraph of $\Gamma$ if for each pair of vertices $a$ and $b$ of $\Gamma^{\prime}, e_{\{a, b\}}$ is $E\left(\Gamma^{\prime}\right)$ whenever $e_{\{a, b\}}$ is in $E(\Gamma)$. We say that $\Gamma_{1}$ has $\Gamma_{2}$ as an induced subgraph (or $\Gamma_{1}$ has induced $\Gamma_{2}$ ) if $\Gamma_{1}$ has an induced subgraph that is an isomorphic copy of $\Gamma_{2}$. Note that for a subset $S$ of the vertex set of a graph $\Gamma$, there is a unique induced subgraph of $\Gamma$ having $S$ as the vertex set. This induced subgraph is called the subgraph generated by $S$ and denoted by $\Gamma_{S}$. It is known that if $\Gamma$ has $\Gamma^{\prime}$ as an induced subgraph, then $C(\Gamma)(A(\Gamma))$ has a subgroup which is isomorphic to $C\left(\Gamma^{\prime}\right)\left(A\left(\Gamma^{\prime}\right)\right)$.

The complement graph $\bar{\Gamma}$ of a graph $\Gamma$ is the graph with $V(\bar{\Gamma})=V(\Gamma)$ and $E(\bar{\Gamma})=\left\{e_{\{a, b\}} \mid e_{\{a, b\}} \notin E(\Gamma)\right\}$. The $n$-cycle, denoted by $C_{n}$, is the graph that is homeomorphic to the circle and has $\mathrm{n}$ edges $(n=3,4,5, \ldots)$. The complement graph $\overline{C_{n}}$ of $C_{n}$ is called the anti n-cycle.

Let $v$ be a vertex in a graph $\Gamma$. The link of $v$ is defined as the set of vertices adjacent to $v$. The link of $v$ is denoted by $\operatorname{link}(v)$. Note that $v$ itself is not in the link of $v$. The star of $v$, denoted by $\operatorname{star}(v)$, is defined as $\{v\} \cup \operatorname{link}(v)$.

For basic facts on graphs, see [6].

Definition $([7,8])$. Let $\Gamma$ be a graph. Let $a, b$ be two different non-adjacent vertices of $\Gamma$. The co-contraction $\widehat{\Gamma}$ of $\Gamma$ relative to $a, b$ is defined to be the graph with $V(\widehat{\Gamma})=(V(\Gamma)-\{a, b\}) \cup\{v\}$ and $E(\widehat{\Gamma})=E\left(\Gamma_{V(\Gamma-\{a, b\})}\right) \cup\left\{e_{\{v, q\}} \mid q \in\right.$ $V(\Gamma)-\{a, b\}$ and $\{a, b\} \subset \operatorname{link}(q)\}$.

If $a$ and $b$ are not adjacent in a graph $\Gamma$, then the co-contraction $\widehat{\Gamma}$ is obtained by collapsing $\{a, b\}$ onto one vertex $v$, removing all the edges connected to $a$ or $b$, and adding new edges $e_{\{v, q\}}$ if $q \in V(\Gamma)-\{a, b\}$ and $q$ is adjacent to both $a$ and $b$ in $\Gamma$. As a matter of fact, what Kim introduced was the cocontraction relative to an anti-connected subset $S$ of $V(\Gamma)$. A subset $S$ of $V(\Gamma)$ is called anti-connected if $\overline{\Gamma_{S}}$ is connected. He was able to prove the following proposition $([7,8])$.

Proposition $2.1([7,8])$. For any co-contraction $\widehat{\Gamma}$ of $\Gamma$, there is a monomorphism from $A(\widehat{\Gamma})$ to $A(\Gamma)$.

Note that the set of two non-adjacent vertices is anti-connected. Kim observed that for a given graph $\Gamma$ and its anti-connected subset $B$, the cocontraction $\widehat{\Gamma}$ of $\Gamma$ relative to $B$ can be obtained from $\Gamma$ by a finite successive sequence of co-contractions relative to two non-adjacent vertices. Therefore, it is enough to deal with the co-contractions relative two non-adjacent vertices 
to prove the above proposition. Likewise, in order to set up a similar result for Coxeter group case, we only need to handle co-contractions relative two non-adjacent vertices.

Recently, Bell made use of Reidemeister-Schreier method to give another proof of Kim's result on co-contractions ([2]). Reidemeister-Schreier method can be found in $[1,2,9,10]$. Let $G$ be a group given by the presentation $\langle X \mid R\rangle$, and suppose $H$ is a subgroup of $G$. Then a useful presentation for $H$ is given by Reidemeister-Schreier method.

Proposition 2.2 (Reidemeister-Schreier method). Let $F$ be free with basis $X$, and let $\pi: F \longrightarrow G$ be the canonical map. Let $P=\pi^{-1}(H)$. And let $T \subset F$ be a right Schreier transversal for $P$ in $F$, i.e., $T$ is a complete set of right coset representatives such that for each element $t$ in $T$ any initial subword of $t$ belongs to $T$ again. Given $w \in F$, let $\bar{w}$ be the unique element of $T$ such that $P w=P \bar{w}$. For each $t \in T$ and $x \in X$, let $s(t, x)=t x(\overline{t x})^{-1}$. Define $S=\{s(t, x) \mid t \in T, x \in X$, and $s(t, x) \neq 1$ in $F\}$. Then $S$ is a basis for the free group $P$. Define a rewriting process $\tau: F \longrightarrow P$ on freely reduced words over $X$ by $\tau\left(y_{1} y_{2} \cdots y_{n}\right)=s\left(1, y_{1}\right) s\left(\overline{y_{1}}, y_{2}\right) \cdots s\left(\overline{y_{1} \cdots y_{n-1}}, y_{n}\right)$, where $y_{i} \in X \cup X^{-1}$. Then $\tau(w)=w \bar{w}^{-1}$ for every freely reduced word $w \in F$, and $H=\langle S| \tau\left(t r t^{-1}\right)=1$ for all $t \in T$ and $\left.r \in R\right\rangle$.

Lemma 2.3 (Bell's lemma [2]). Suppose $A(\Gamma)$ is a right-angled Artin group, and let $n$ be a positive integer. Choose a vertex $z \in V(\Gamma)$, and define $\phi$ : $A(\Gamma) \longrightarrow\left\langle x \mid x^{n}=1\right\rangle$ by $\phi(z)=x$ and $\phi(v)=1$ if $v \neq z$. Then $\operatorname{ker} \phi$ is the right-angled Artin group whose defining graph $\Gamma^{\prime}$ is obtained by gluing $n$ copies of $\Gamma-\operatorname{star}(z)$ to star $(z)$ along $\operatorname{link}(z)$. Moreover, the vertices of $\Gamma^{\prime}$ naturally correspond to the following generating set.

$$
\begin{aligned}
\left\{z^{n}\right\} \cup \operatorname{link}(z) \cup\{u \mid u \notin \operatorname{star}(z)\} \cup\left\{z u z^{-1} \mid u \notin \operatorname{star}(z)\right\} \cup \cdots \\
\cup\left\{z^{n-1} u z^{1-n} \mid u \notin \operatorname{star}(z)\right\} .
\end{aligned}
$$

Bell's method can also be applied to right-angled Coxeter groups to obtain the following proposition.

Proposition 2.4. Suppose $C(\Gamma)$ is a right-angled Coxeter group. Choose a vertex $z \in V(\Gamma)$, and define $\phi: C(\Gamma) \longrightarrow\left\langle x \mid x^{2}=1\right\rangle \cong \mathbb{Z}_{2}$ by $\phi(z)=x$ and $\phi(v)=1$ if $v \neq z$. Then $\operatorname{ker} \phi$ is the right-angled Coxeter group whose defining graph $\Gamma^{\prime \prime}$ is the double of $\Gamma-\{z\}$ along $\operatorname{link}(z)$. Moreover, the vertices of $\Gamma^{\prime \prime}$ naturally correspond to the following generating set.

$$
\operatorname{link}(z) \cup\{u \mid u \notin \operatorname{star}(z)\} \cup\left\{z u z^{-1} \mid u \notin \operatorname{star}(z)\right\} .
$$

Proof. Let $G=C(\Gamma)$ and $F$ be free on $X=V(\Gamma)$ and let $R$ be the set of relators of $C(\Gamma)$. We apply Proposition 2.2 to get a proper presentation for ker $\phi$. Let $P$ be the inverse image of $\operatorname{ker} \phi$ in $F$ under the canonical map $F \longrightarrow G$. It is easy to see that the set $T=\{1, z\}$ is a right Schreier transversal for $P$ in $F$. In order to find the generating set in Reidemeister-Schreier method, we have 
to examine whether each of the following elements $s(1, v), s(1, z), s(z, v), s(z, z)$ with $v \neq z$ is trivial or not. Note that

$$
\begin{aligned}
& s(1, v)=v \bar{v}^{-1}=v \text { if } v \neq z, \\
& s(z, v)=z v \overline{z v}^{-1}=z v z^{-1} \text { if } v \neq z, \\
& s(1, z)=z \bar{z}^{-1}=1, \\
& s(z, z)=z^{2} \bar{z}^{-1}=z^{2} .
\end{aligned}
$$

Thus the generating set of ker $\phi$ we look for is the set $\{s(z, z), s(1, v), s(z, v) \mid v \in$ $V(\Gamma)-\{z\}\}$. Next, to identify the relators, we need to compute $\tau\left(t r t^{-1}\right)$ for each $t \in T$ and $r \in R$. It is straightforward to compute the following:

$$
\begin{gathered}
\tau\left(v^{2}\right)=v^{2} \bar{v}^{-1}=v^{2}=s(1, v)^{2} \text { if } v \neq z \\
\tau\left(z v^{2} z^{-1}\right)=z v^{2} z^{-1} \overline{z v^{2} z^{-1}}=z v^{2} z^{-1}=s(z, v)^{2} \text { if } v \neq z, \\
\tau\left(z^{2}\right)=z^{2} \bar{z}^{2}-1=z^{2}=s(z, z), \\
\tau\left(z z^{2} z^{-1}\right)=z^{2} \bar{z}^{-1}=z^{2}=s(z, z), \\
\tau([u, v])=[u, v] \overline{[u, v]}=[u, v]=[s(1, u), s(1, v)] \\
\text { if } u, v \neq z \text { and } u, v \text { areadjacent in } \Gamma, \\
\tau\left(z[u, v] z^{-1}\right)=z[u, v] z^{-1} \overline{z[u, v] z^{-1}-1}=z[u, v] z^{-1}=[s(z, u), s(z, v)] \\
\text { if } u, v \neq z \operatorname{and} u, v \text { are adjacent in } \Gamma, \\
\tau([z, w])=[z, w][z, w]=[z, w]=s(z, w) s(1, w)^{-1} \\
\text { if } w \in \operatorname{link}(z) \text { in } \Gamma, \\
\tau\left(z[z, w] z^{-1}\right)=z[z, w] z^{-1} \overline{z[z, w] z^{-1}}-1=z[z, w] z^{-1}=z^{2} w z^{-1} w^{-1} z^{-1} \\
=z^{2} w z^{-2} z w^{-1} z^{-1} \\
=s(z, z) s(1, w) s(z, z)^{-1} s(z, w)^{-1} \\
\text { if } w \in \operatorname{link}(z) \text { in } \Gamma .
\end{gathered}
$$

Since $s(z, z)=\tau\left(z^{2}\right), s(z, z)=1$ in $\operatorname{ker} \phi$. Thus, the last equality implies that $s(1, w)=s(z, w)$ in $\operatorname{ker} \phi$ if $w \in \operatorname{link}(z)$. Therefore, $\operatorname{ker} \phi$ has the presentation in which generators are $s(1, v), s(z, v)$ with $v \in V(\Gamma)-\{z\}\}$, and relators are $s(1, v)^{2}, s(z, v)^{2},\left[s\left(1, u_{1}\right), s\left(1, u_{2}\right)\right],\left[s\left(z, u_{1}\right), s\left(z, u_{2}\right)\right], s(z, w) s(1, w)^{-1}$ where $v, u_{1}, u_{2} \neq z$, and $u_{1}, u_{2}$ are adjacent, and $w \in \operatorname{link}(z)$. The conclusion in the proposition follows.

By using Lemma 2.3, Bell gave another proof of Proposition 2.1. Likewise, Proposition 2.4 gives rise to a similar result for right-angled Coxeter group.

Theorem 2.5. For any co-contraction $\widehat{\Gamma}$ of $\Gamma$, there is a monomorphism from $C(\widehat{\Gamma})$ to $C(\Gamma)$. 
Proof. Let $a$ and $b$ be two non-adjacent vertices on $\Gamma$. And let $\widehat{\Gamma}$ be the cocontraction of $\Gamma$ relative to $a$ and $b$. Let $v_{a, b}$ be the new vertex in $\widehat{\Gamma}$. Consider the homomorphism $\phi: C(\Gamma) \longrightarrow\left\langle x \mid x^{2}=1\right\rangle \cong \mathbb{Z}_{2}$ by $\phi(a)=x$ and $\phi(v)=1$ if $v \neq a$. By Proposition 2.4, $\operatorname{ker} \phi \leq C(\Gamma)$ is the right-angled Coxeter group $C\left(\Gamma^{\prime \prime}\right)$ whose defining graph $\Gamma^{\prime \prime}$ is the double of $\Gamma-\{a\}$ along $\operatorname{link}(a)$. It is well-known that if $\Gamma_{1}$ is an induced subgraph of $\Gamma_{2}$, then $C\left(\Gamma_{1}\right)$ is isomorphic to a subgroup of $C\left(\Gamma_{2}\right)$. Therefore, it suffices to show that $\Gamma^{\prime \prime}$ has an induced subgraph which is isomorphic to $\widehat{\Gamma}$. Let $\Gamma^{\prime}$ be a copy of $\Gamma$ and $a^{\prime}$ be the corresponding vertex to $a$ in $\Gamma^{\prime}$. Then $\Gamma^{\prime \prime}$ can be regarded as the graph amalgamation of the induced subgraph generated by $V(\Gamma)-\{a\}$ in $\Gamma$ and the induced subgraph generated by $V\left(\Gamma^{\prime}\right)-\left\{a^{\prime}\right\}$ in $\Gamma^{\prime}$ along $\operatorname{link}(a)=\operatorname{link}\left(a^{\prime}\right)$. For each $v \in V(\Gamma)-\operatorname{star}(a) \subset V\left(\Gamma^{\prime \prime}\right)$, we denote the corresponding vertex of $v$ in the copy $V\left(\Gamma^{\prime}\right)-\operatorname{star}\left(a^{\prime}\right)$ by $v^{\prime}$. Note that $b \in V(\Gamma)-\operatorname{star}(a)$ and so $b^{\prime} \in V\left(\Gamma^{\prime}\right)-\operatorname{star}\left(a^{\prime}\right)$ in $V\left(\Gamma^{\prime \prime}\right)$. Consider the induced subgraph $T$ of $\Gamma^{\prime \prime}$ generated by $V(T):=(V(\Gamma)-\{a, b\}) \cup\left\{b^{\prime}\right\}$. Let $w \in V(T)-\left\{b^{\prime}\right\} . w$ is adjacent to $b^{\prime}$ in $T$ if and only if $w \in \operatorname{link}(a)$ and $w$ is adjacent to $b$ in $\Gamma$, that is, $w$ is adjacent to both $a$ and $b$ in $\Gamma$. It is easy to see that $T$ is isomorphic to $\widehat{\Gamma}$. In fact, sending $b^{\prime}$ to $v_{a, b}$ and $w$ to $w$ for $w \neq a, b$ defines an isomorphism $T \longrightarrow \widehat{\Gamma}$.

\section{Negative examples for Gordon, Long, and Reid's question}

By a hyperbolic surface group, we mean the fundamental group of a closed orientable surface of genus $n$ for some $n \geq 2$. Let $\operatorname{Isom}\left(H^{2}\right)$ denote the group of isometries of $H^{2}$. Consider a regular $n$-gon in $H^{2}$ with all its vertex angles equal to $\frac{\pi}{2}$. It is known that the subgroup of $\operatorname{Isom}\left(H^{2}\right)$ generated by reflections in the sides of the regular $n$-gon is isomorphic to the right-angled Coxeter group $C\left(C_{n}\right)$ ([5]). In [12], Scott showed that the subgroup of $\operatorname{Isom}\left(H^{2}\right)$ generated by reflections in the sides of the regular pentagon contains a subgroup of index four which is isomorphic to the fundamental group of the non-orientable closed surface with Euler number -1 . Therefore, we see that $C\left(C_{5}\right)$ contains a subgroup of index eight which is isomorphic to the fundamental group of the orientable closed surface of genus $2([11])$. Using co-contraction, it can be seen that for each $n \geq 5$ the right-angled Coxeter group $C\left(C_{n}\right)$ contains $C\left(C_{5}\right)$, and so $C\left(C_{n}\right)$ contains a hyperbolic surface subgroup. Therefore for any graph $\Gamma$ containing an induced $n$-cycle, $C(\Gamma)$ contains a hyperbolic surface group. In [5], Gordon, Long, and Reid asked if the converse holds while they proved that a word-hyperbolic (not necessarily right-angled) Coxeter group either is torsion-free or contains a hyperbolic surface group.

Question 3.1 ([5]). Does a right-angled Coxeter group $C(\Gamma)$ contain a hyperbolic surface subgroup if and only if $\Gamma$ contains an induced $n$-cycle for some $n \geq 5$ ? 
In this section, we build up examples which provide a negative answer to this question using Theorem 2.5. First, note that for each $n \geq 3$, the anti $n$-cycle $\overline{C_{n}}$ is the co-contraction of $\overline{C_{n+1}}$ relative to a pair of non-adjacent vertices, which is adjacent in $C_{n+1}$, and so $C\left(\overline{C_{n}}\right)$ is isomorphic to a subgroup of $C\left(\overline{C_{n+1}}\right)$. Since the 5 -cycle is equal to its complement graph, that is, $C_{5}=\overline{C_{5}}, C\left(\overline{C_{n}}\right)$ contains $C\left(C_{5}\right)$ as a subgroup for each $n \geq 5$. In fact, it is easy to see that $C\left(C_{5}\right)=C\left(\overline{C_{5}}\right) \leq C\left(\overline{C_{6}}\right) \leq C\left(\overline{C_{7}}\right) \leq \cdots$. Therefore, if $\Gamma$ contains an induced $C_{n}$ or $\overline{C_{n}}$ for some $n \geq 5$, then $C(\Gamma)$ contains a hyperbolic surface subgroup.

Proposition 3.2. If $\Gamma$ contains an induced $C_{n}$ or $\overline{C_{n}}$ for some $n \geq 5$, then $C(\Gamma)$ contains a hyperbolic surface subgroup.

For $n \geq 6, \overline{C_{n}}$ does not contain induced $C_{m}, m \geq 5$. In fact, $C_{n}$ does not contain an induced $\overline{C_{m}}$ as every vertex of $\overline{C_{m}}$ has valence greater than 2 if $m \geq 5$. It can be seen easily that a graph $\Gamma_{1}$ is an induced subgraph of a graph $\Gamma_{2}$ if and only if $\overline{\Gamma_{1}}$ is an induced subgraph of $\overline{\Gamma_{2}}$. Hence $\overline{C_{n}}$ does not contain an induced $C_{m}$. It follows that $C\left(\overline{C_{n}}\right)(n \geq 6)$ is a right-angled Coxeter group containing a hyperbolic surface group, while its underlying graph does not contain any induced $n$-cycle for $n \geq 5$.

Corollary 3.3. There is a right-angled Coxeter group $C(\Gamma)$ containing a hyperbolic surface subgroup, while its underlying graph $\Gamma$ does not contain any induced $n$-cycle for $n \geq 5$.

This corollary gives a negative answer to Question 3.1. It is known that for each $n \geq 5$ the right-angled Artin group $A\left(C_{n}\right)$ contains a hyperbolic surface subgroup ([13]). The above argument about anti $n$-cycles and their co-contractions was originally used by $\operatorname{Kim}[7,8]$ to prove the following proposition.

Proposition $3.4([7,8])$. If $\Gamma$ contains an induced $C_{n}$ or $\overline{C_{n}}$ for some $n \geq 5$, then $A(\Gamma)$ contains a hyperbolic surface subgroup.

This proposition answered negatively to the right-angle Artin group version of Question 3.1, which was also raised by Gordon, Long, and Reid in [5]: Does a right-angled Artin group $A(\Gamma)$ contain a hyperbolic surface subgroup if and only if $\Gamma$ contains an induced $n$-cycle for some $n \geq 5$ ?

It might be interesting to give different kinds of examples which justifies Corollary 3.3. We use the results of Kim [7, 8] and Davis, Januszkiewicz [4] to construct such examples. Given a graph $\Gamma$, construct a graph $\Gamma^{\prime \prime}$ with the vertex set $V\left(\Gamma^{\prime \prime}\right)=V(\Gamma) \times\{0,1\}$ in the following way:

- Two vertices $(a, 1),(b, 1)$ in $V(\Gamma) \times\{0,1\}$ are connected by an edge in $\Gamma^{\prime \prime}$ if and only if $(a, b) \in E(\Gamma)$.

- $V(\Gamma) \times\{0\}$ forms a complete subgraph in $\Gamma^{\prime \prime}$.

- $(a, 0)$ and $(b, 1)$ are connected by an edge if and only if $a \neq b$. 
Davis and Januszkiewicz [4] showed that $A(\Gamma)$ is a normal subgroup of index $2^{|V(\Gamma)|}$ in $C\left(\Gamma^{\prime \prime}\right)$. So if $A(\Gamma)$ contains a hyperbolic surface subgroup, then $C\left(\Gamma^{\prime \prime}\right)$ contains a hyperbolic surface subgroup.

Let $n \geq 6$. We claim that $\Gamma^{\prime \prime}$ does not contain an induced $m$-cycle for all $m \geq 5$, when $\Gamma=\overline{C_{n}}$. Suppose not. Let $C_{m}$ be an induced $m$-cycle for some $m$ in $\Gamma^{\prime \prime}$. Since $\overline{C_{n}}$ with $n \geq 6$ does not contain an induced $m$-cycle for any $m \geq 5$, there must be at least one vertex of $C_{m}$ in $V(\Gamma) \times\{0\}$. But since $V(\Gamma) \times\{0\}$ forms a complete subgraph in $\Gamma^{\prime \prime}$, there can be at most two vertices of $C_{m}$ in $V(\Gamma) \times\{0\}$.

First, suppose there are two vertices of $C_{m}$ in $V(\Gamma) \times\{0\}$. Let $(A, 0)$ and $(B, 0)$ be such points. Since $m \geq 5$, there are at least three vertices of $C_{m}$ in $V(\Gamma) \times\{1\}$. It follows that there is a vertex $(P, 1)$ of $C_{m}$ in $V(\Gamma) \times\{1\}$ with $P \neq A, B$. The way we construct $\Gamma^{\prime \prime}$ forces the induced cycle $C_{m}$ to contain a 3 -cycle whose vertices are $(A, 0),(B, 0)$ and $(P, 1)$. For the case where there is only one vertex $(A, 0)$ of $C_{m}$ in $V(\Gamma) \times\{0\}$, note that there should be more than three vertices of $C_{m}$ in $V(\Gamma) \times\{1\}$, since $m \geq 5$. Among these vertices, we can choose three vertices $(P, 1),(Q, 1),(R, 1)$ with $A \neq P, Q, R$, which means the vertex $(A, 0)$ has valence greater than 2 in $C_{m}$. This is a contradiction. This proves our claim.

Proposition 3.4 says that the right-angled Artin group $A\left(\overline{C_{n}}\right)(n \geq 5)$ contains a hyperbolic surface subgroup, thereby $C\left({\overline{C_{n}}}^{\prime \prime}\right)$ has a hyperbolic surface subgroup although $\overline{C_{n}}$ " does not contain any induced $m$-cycle for $m \geq 5$. This justifies Corollary 3.3 again.

Now we describe another example which ensures Corollary 3.3 is true. Given a graph $\Gamma$, we define a graph $\Gamma^{\prime}$ as follows: The vertex set of $\Gamma^{\prime}$ is $V(\Gamma) \times\{-1,1\}$. The vertices $(a,-1),(b, 1)$ in $V(\Gamma) \times\{-1,1\}$ are connected by an edge in $\Gamma^{\prime}$ if and only if $a \neq b$ and $(a, b) \in E(\Gamma)$. Davis and Januszkiewicz [4] showed that $C\left(\Gamma^{\prime}\right)$ is a normal subgroup of index $2^{|V(\Gamma)|}$ in $C\left(\Gamma^{\prime \prime}\right)$.

Proposition 3.5. $A(\Gamma)$ contains a hyperbolic surface subgroup if and only if $C\left(\Gamma^{\prime}\right)$ contains a hyperbolic surface subgroup.

Proof. It is known that any finite index subgroup of a hyperbolic surface group is isomorphic to a hyperbolic surface group. Suppose that $A(\Gamma)$ contains a hyperbolic surface subgroup $Q$. Then $C\left(\Gamma^{\prime \prime}\right)$ contains a hyperbolic surface subgroup $Q$. Since $C\left(\Gamma^{\prime}\right)$ is a finite index subgroup of $C\left(\Gamma^{\prime \prime}\right), C\left(\Gamma^{\prime}\right) \cap Q$ is a finite index subgroup of $Q$, so is isomorphic to a hyperbolic surface subgroup. Hence, $C\left(\Gamma^{\prime}\right)$ contains a hyperbolic surface subgroup. The same argument holds for the converse, as $A(\Gamma)$ is a finite index normal subgroup of $C\left(\Gamma^{\prime \prime}\right)$

An argument similar to the case of $\overline{C_{n}}$ ' shows that ${\overline{C_{n}}}^{\prime}$ with $n \geq 6$ does not contain an induced $m$-cycle for all $m \geq 5$. But $C\left({\overline{C_{n}}}^{\prime}\right)$ contains a hyperbolic surface subgroup. 
CO-CONTRACTIONS OF GRAPHS AND RIGHT-ANGLED COXETER GROUPS 1065

\section{References}

[1] G. Baumslag, Topics in Combinatorial Group Theory, Lectures in Mathematics ETH Zürich, Birkhäuser Verlag, Basel, 1993.

[2] R. W. Bell. Combinatorial methods for detecting surface subgroups in right-angled Artin groups, arxiv.org/1012.4208.

[3] R. Charney, An introduction to right-angled Artin groups, Geom. Dedicata 125 (2007), 141-158.

[4] M. W. Davis and T. Januszkiewicz, Right-angled Artin groups are commensurable with right-angled Coxeter groups, J. Pure Appl. Algebra 153 (2000), no. 3, 229-235.

[5] C. McA. Gordon, D.D. Long, and A. W. Reid, Surface subgroups of Coxeter and Artin groups, J. Pure Appl. Algebra 189 (2004), no. 1-3, 135-148.

[6] J. Gross and T. W. Tucker, Topological Graph Theory, A Wiley-Interscience Publication, John Wiley and Sons, 1987.

[7] S. Kim, Hyperbolic Surface Subgroups of Right-Angled Artin Groups and Graph Products of Groups, PhD thesis, Yale University, 2007.

[8] _ Co-contractions of graphs and right-angled Artin groups, Algebr. Geom. Topol. 8 (2008), no. 2, 849-868.

[9] R. C. Lyndon and P. E. Schupp, Combinatorial Group Theory, Springer, Berlin, Heidelberg, New York, 1977.

[10] W. Magnus, A. Karrass, and D. Solitar, Combinatorial Group Theory, Dover Publications Inc., 1976.

[11] W. Massey, Algebraic Topology: An Introduction, GTM 56, Springer, 1977.

[12] P. Scott, Subgroups of surface groups are almost geometric, J. London Math. Soc. (2) 17 (1978), no. 3, 555-565.

[13] H. Servatius, C. Droms, and B. Servatius, Surface subgroups of graph group, Proc. Amer. Math. Soc. 106 (1989), no. 3, 573-578.

JONGTAE KIM

Department of Mathematics

KONKUK UNIVERSITY

SEOUL 143-701, KoreA

E-mail address: dkgurel@hammail.net

MyOungho MoON

Department of Mathematics Education

KONKUK UNIVERSITY

SEOUl 143-701, KoreA

E-mail address: mhmoon@konkuk.ac.kr 